\title{
SUSY effects in Kaon physics: Lepton Universality tests and rare decays
}

\section{Paride Paradisi*}

Technical University Munich, Physics Department, D-85748 Garching, Germany,

E-mail: paride.paradisi@ph.tum.de

Deviations from the Standard Model (SM) expectations of the Lepton Universality (LU) breaking represent a powerful tool to probe New Physics (NP) effects. In the present document, we focus on $R_{K}=\Gamma\left(K_{e 2}\right) / \Gamma\left(K_{\mu 2}\right)$, stressing the importance of $R_{K}$ measurements with an accuracy at the \% level to probe lepton-flavor violating effects arising in Supersymmetric (SUSY) theories. Finally, we also review the role of FCNC rare $K$ decays in shedding light on NP.

2009 KAON International Conference

June 09 - 12, 2009

Tsukuba, Japan 


\begin{tabular}{lc}
\hline \hline & $R_{K}^{e / \mu}\left[10^{-5}\right]$ \\
\hline SM prediction [1] & $2.477 \pm 0.001$ \\
\hline PDG 2006 [2] & $2.45 \pm 0.11$ \\
\hline KLOE [3] & $2.493 \pm 0.025 \pm 0.019$ \\
\hline NA48/2 '07 prel. [4] & $2.500 \pm 0.016$ \\
\hline \hline
\end{tabular}

Figure 1: Current experimental data on $R_{K}^{e / \mu}$.

\section{Introduction}

High precision electroweak tests, such as deviations from the SM expectations of the LFU breaking, represent a powerful tool to probe the SM and, hence, to constrain or obtain indirect hints of new physics beyond it. Kaon and pion physics are obvious grounds where to perform such tests, for instance in the $\pi \rightarrow \ell v_{\ell}$ and $K \rightarrow \ell v_{\ell}$ decays, where $\ell=e$ or $\mu$. In particular, the ratios

$$
R_{P}^{e / \mu}=1+\Delta r_{P}^{e / \mu}=\frac{\mathscr{B}(P \rightarrow \mu v)}{\mathscr{B}(P \rightarrow e v)}
$$

can be predicted with excellent accuracies in the SM, both for $P=\pi(0.02 \%$ accuracy [1]) and $P=K(0.04 \%$ accuracy [1]), allowing for some of the most significant tests of LFU. These precision tests are equally interesting and fully complementary to the flavour-conserving electroweak precisions tests and to the FCNC tests performed at $B$ factories: the smallness of NP effects is more than compensated by the excellent experimental resolution and the good theoretical control.

The limiting factor in the determination $R_{K}^{e / \mu}$ is the $K \rightarrow e v$ rate, whose experimental knowledge has been quite poor so far.

The current world average composed of the recent KLOE result [3] $R_{K}^{e / \mu}=(2.493 \pm 0.025 \pm$ $0.019) \times 10^{-5}$ and the three 1970s measurements [2] is $R_{K}^{e / \mu}=(2.467 \pm 0.024) \times 10^{-5}$ (see Fig. 1). It has $1 \%$ relative precision and is compatible with the SM.

The NA62 experiment at CERN collected a large sample of $K^{+} \rightarrow e^{+} v$ decays during a dedicated run in 2007. The preliminary result of the analysis of a partial data sample of 51089 $K^{+} \rightarrow e^{+} v$ candidates is $R_{K}^{e / \mu}=(2.500 \pm 0.016) \times 10^{-5}$ [4], consistent with the SM expectation. Exploiting the full 2007-08 data set, the NA62 experiment at CERN aims to reach the precision of $0.4 \%$ for $R_{K}$.

In the following, we consider low-energy minimal SUSY extensions of the SM (MSSM) with $\mathrm{R}$ parity as the source of new physics to be tested by $R_{K}^{e / \mu}$ [5]. The question we intend to address is whether SUSY can cause deviations from $\mu-e$ universality in $K_{l 2}$ at a level which can be probed with the present attained experimental sensitivity, namely at the percent level. We will show that i) it is indeed possible for regions of the MSSM to obtain $\Delta r_{N P}^{e / \mu}$ of $\mathscr{O}\left(10^{-2}\right)$ and ii) such large contributions to $K_{\ell 2}$ do not arise from SUSY lepton flavor conserving (LFC) effects, but, rather, from LFV ones. 


\section{Lepton Universality in $K \rightarrow \ell v$}

Due to the V-A structure of the weak interactions, the SM contributions to $K_{\ell 2}$ are helicity suppressed; hence, these processes are very sensitive to non-SM effects (such as multi-Higgs effects) which might induce an effective pseudoscalar hadronic weak current. In particular, charged Higgs bosons $\left(H^{ \pm}\right)$appearing in any model with two Higgs doublets (including the SUSY case) can contribute at tree level to the above processes. The tree level partial width is given by [6]:

$$
\frac{\Gamma\left(K^{-} \rightarrow \ell^{-} \bar{v}\right)}{\Gamma\left(K^{-} \rightarrow \ell^{-} \bar{v}\right)_{S M}}=r_{K}=\left[1-\tan ^{2} \beta\left(\frac{m_{K}^{2}}{m_{H^{ \pm}}^{2}}\right)\right]^{2},
$$

Eq. (2.1) clearly show that a tree level contribution doesn't introduce any lepton flavour dependent correction. The first SUSY contributions violating the $\mu-e$ universality in $K \rightarrow \ell v$ decays arise at the one-loop level with various diagrams involving exchanges of (charged and neutral) Higgs scalars, charginos, neutralinos and sleptons. For our purpose, it is relevant to divide all such contributions into two classes: i) LFC contributions where the charged meson M decays without FCNC in the leptonic sector, i.e. $K \rightarrow \ell v_{\ell}$; ii) LFV contributions $K \rightarrow \ell_{i} v_{k}$, with $i$ and $k$ referring to different generations (in particular, the interesting case will be for $i=e, \mu$, and $k=\tau$ ).

\subsection{The lepton flavour conserving case}

One-loop corrections to $R_{K}$ include box, wave function renormalization and vertex contributions from SUSY particle exchange. The dominant diagrams containing one loop corrections to the $l W v_{l}$ vertex have the following suppression factors (compared to the tree level graph) [5]

$$
\Delta r_{K}^{e / \mu} \sim \frac{g_{2}^{2}}{16 \pi^{2}} \frac{m_{W}^{2}}{M_{S U S Y}^{2}}\left(\frac{m_{\tilde{\ell}_{\mu}}^{2}-m_{\tilde{\ell}_{e}}^{2}}{m_{\tilde{\ell}_{\mu}}^{2}+m_{\tilde{\ell}_{e}}^{2}}\right)
$$

for loops generated by charginos/neutralinos and sleptons. Even if we assume $\mathscr{O}(1)$ a quite large mass splitting among slepton masses we end up with $r_{K}^{e / \mu} \leq 10^{-4}$.

In conclusion, SUSY effects with flavor conservation in the leptonic sector can differently contribute to the $K \rightarrow e v_{e}$ and $K \rightarrow \mu v_{\mu}$ decays, hence inducing a $\mu-e$ non-universality in $R_{K}$, however such effects are still orders of magnitude below the level of the present experimental sensitivity on $R_{K}$. The same conclusions hold for $R_{\pi}$.

\subsection{The lepton flavour violating case}

It is well known that models containing at least two Higgs doublets generally allow flavour violating couplings of the Higgs bosons with the fermions. In the MSSM such LFV couplings are absent at tree level. However, once non holomorphic terms are generated by loop effects (so called HRS corrections [7]) and given a source of LFV among the sleptons, Higgs-mediated (radiatively induced) $H \ell_{i} \ell_{j}$ LFV couplings are unavoidable [8].

It has been shown [5] that Higgs-mediated LFV couplings generate a breaking of the $\mu-e$ universality in the purely leptonic $K^{ \pm}$decay. 
One could naively think that SUSY effects in the LFV channels $K \rightarrow \ell_{i} v_{k}$ are further suppressed with respect to the LFC ones. On the contrary, charged Higgs mediated SUSY LFV contributions, in particular in the kaon decays into an electron or a muon and a tau neutrino, can be strongly enhanced.

The quantity which now accounts for the deviation from the $\mu-e$ universality reads:

$$
R_{K}^{L F V}=\frac{\sum_{i} \Gamma\left(K \rightarrow e v_{i}\right)}{\sum_{i} \Gamma\left(K \rightarrow \mu v_{i}\right)} \quad i=e, \mu, \tau
$$

with the sum extended over all (anti)neutrino flavors (experimentally one determines only the charged lepton flavor in the decay products).

The dominant SUSY contributions to $R_{K}^{L F V}$ arise from the charged Higgs exchange. The effective LFV Yukawa couplings we consider are

$$
\ell H^{ \pm} v_{\tau} \rightarrow \frac{g_{2}}{\sqrt{2}} \frac{m_{\tau}}{M_{W}} \Delta_{R}^{3 l} \tan ^{2} \beta \quad \ell=e, \mu
$$

A crucial ingredient for the effects we are going to discuss is the quadratic dependence on $\tan \beta$ in the above coupling: one power of $\tan \beta$ comes from the trilinear scalar coupling in Fig.1, while the second one is a specific feature of the above HRS mechanism.

The LFV $\Delta_{R}^{3 \ell}$ parameters are induced at one loop level and it turns out that $\Delta_{R}^{3 \ell} \leq 10^{-3}$ [5]. Making use of the LFV Yukawa coupling in Eq. (2.3), it turns out that the dominant contribution to $\Delta r_{N P}^{e-\mu}$ reads [5]:

$$
R_{K}^{L F V} \simeq R_{K}^{S M}\left[1+\left(\frac{m_{K}^{4}}{M_{H}^{4}}\right)\left(\frac{m_{\tau}^{2}}{m_{e}^{2}}\right)\left|\Delta_{R}^{31}\right|^{2} \tan ^{6} \beta\right]
$$

In Eq. (2.4) terms proportional to $\Delta_{R}^{32}$ are neglected given that they are suppressed by a factor $m_{e}^{2} / m_{\mu}^{2}$ with respect to the term proportional to $\Delta_{R}^{31}$.

Taking $\Delta_{R}^{31} \simeq 5 \cdot 10^{-4}, \tan \beta=40$ and $M_{H}=500 \mathrm{GeV}$ we end up with $R_{K}^{L F V} \simeq R_{K}^{S M}(1+0.013)$. We see that in the large (but not extreme) $\tan \beta$ regime and with a relatively heavy $H^{ \pm}$, it is possible to reach contributions to $\Delta r_{K S U S Y}^{e-\mu}$ at the percent level thanks to the possible LFV enhancements arising in SUSY models.

In Fig. 2, on the left, we report $\Delta r_{K}^{e / \mu}$ as a function of $\mathscr{B}(\tau \rightarrow e \gamma)$ and $\mathscr{B}(\tau \rightarrow e \eta)$ while, on the right, we report $\Delta r_{K}^{e / \mu}$ as a function of $M_{H}$. The plots have been obtained by means of a scan over the following parameter space: $\left(m_{L, R}, m_{\tilde{Q}}, m_{\tilde{g}}, m_{\tilde{W}}, m_{\tilde{B}}, M_{H}\right)<2.5 \mathrm{TeV}, \mu<5 \mathrm{TeV},\left|\delta_{R R}\right|<0.5$, $\left|\delta_{L L}\right|=0$ and $\tan \beta<60$ and imposing all the constraints discussed in [5]. Black dots refer to the points satisfying the $(g-2)_{\mu}$ anomaly at the $95 \%$ C.L., i.e. $1 \times 10^{-9}<\Delta a_{\mu}<5 \times 10^{-9}$. Fig. 2 clearly shows that there are quite a lot of points in the interesting region where $0.001<\Delta r_{K}^{e / \mu}<0.01$ accounting for the $(g-2)_{\mu}$ anomaly and that are compatible with the experimental constraints of $\mathscr{B}(\tau \rightarrow e \gamma)$ and $\mathscr{B}(\tau \rightarrow e \eta)$.

We also emphasize that experimentally visible effects in $\Delta r_{K}^{e / \mu}$ (at the $0.1 \%$ level) can be reached up to charged Higgs masses at the TeV scale, as shown in Fig. 2 on the right. Moreover, we also stress that the present experimental bounds on $\Delta r_{K}^{e / \mu}$ at the $\%$ level already set constraints on the SUSY parameter space. 

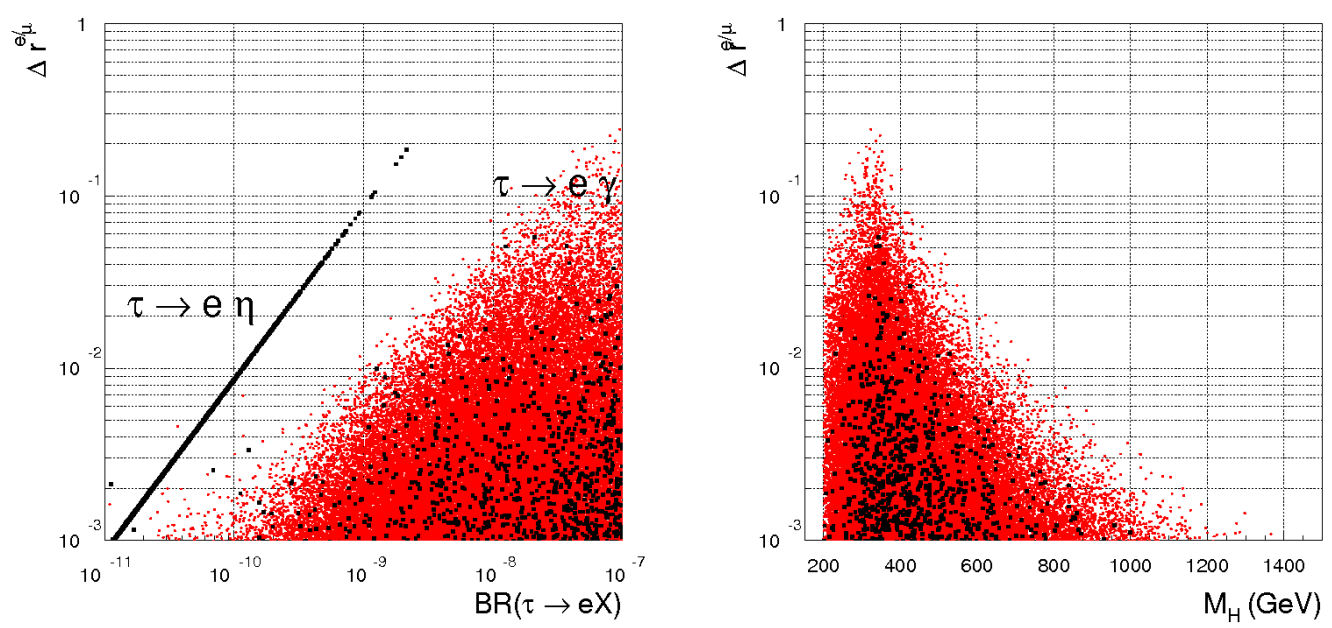

Figure 2: $\quad$ Left: $\Delta r_{K}^{e / \mu}$ as a function of $\mathscr{B}(\tau \rightarrow e \gamma)$ and $\mathscr{B}(\tau \rightarrow e \eta)$. Right: $\Delta r_{K}^{e / \mu}$ as a function of $M_{H}$. Both plots have been obtained by means of a scan over the following parameter space: $\left(m_{L, R}, m_{\tilde{Q}}, m_{\tilde{g}}, m_{\tilde{W}}, m_{\tilde{B}}, M_{H}\right)<2.5 \mathrm{TeV}, \mu<5 \mathrm{TeV},\left|\delta_{R R}\right|<0.5,\left|\delta_{L L}\right|=0$ and $\tan \beta<60$. Black dots refer to the points satisfying $1 \times 10^{-9}<(g-2)_{\mu}<5 \times 10^{-9}$.

Turning to pion physics, one could wonder whether the analogous quantity $\Delta r_{\pi S U S Y}^{e-\mu}$ is able to constrain SUSY LFV. However, the correlation between $\Delta r_{\pi S U S Y}^{e-\mu}$ and $\Delta r_{K S U S Y}^{e-\mu}$ :

$$
\Delta r_{\pi S U S Y}^{e-\mu} \simeq\left(\frac{m_{d}}{m_{u}+m_{d}}\right)^{2}\left(\frac{m_{\pi}^{4}}{m_{k}^{4}}\right) \Delta r_{K S U S Y}^{e-\mu}
$$

clearly shows that the constraints on $\Delta r_{K s u s y}^{e-\mu}$ force $\Delta r_{\pi s u s y}^{e-\mu}$ to be much below its actual experimental upper bound.

A key ingredient for the generation of LFU breaking effects are large $\tan \beta$ values so, it is legitimate to ask how natural this framework is.

In this respect, we remind that values of $\tan \beta \sim 30-50$ can allow the unification of top and bottom Yukawa couplings, as predicted in well-motivated grand-unified models [9]. Moreover, from a low energy point of view, large $\tan \beta$ values lead to interesting phenomenological virtues [10]: the present $(g-2)_{\mu}$ anomaly and the upper bound on the Higgs boson mass can be easily accommodated, while satisfying all the present tight constraints in the electroweak and flavor sectors. Additional low-energy signatures of this scenario could possibly show up in the near future in $\mathscr{B}(B \rightarrow \tau v)$ and $\mathscr{B}\left(B_{s, d} \rightarrow \ell^{+} \ell^{-}\right)$. Additionally, in the regime with large $\tan \beta$, the relic-density constraints can be easily satisfied mainly in the so called $A$-funnel region [11].

\section{Rare $K$ decays}

FCNC are among the most powerful windows into physics beyond the SM. Indeed, they are generated only at the quantum level therefore they are particularly sensitive to electroweak scale physics. As a result, if NP occurs at a scale not much above the TeV scale, we expect significant deviations with respect to the SM expectations. 


\begin{tabular}{|c|c|c|c|}
\hline Channel & $\begin{array}{c}\text { short-distance } \\
\text { contribution } \\
\text { (rate \%) }\end{array}$ & $\begin{array}{c}\text { irreducible } \\
\text { th. error on } \\
\text { s.d. contrib. }\end{array}$ & $\begin{array}{c}\text { SM BR } \\
\text { (central } \\
\text { value) }\end{array}$ \\
\hline$K_{L} \rightarrow \pi^{0} v \bar{v}$ & $>99 \%$ & $\sim 1 \%$ & $3 \times 10^{-11}$ \\
\hline$K^{+} \rightarrow \pi^{+} v \bar{v}$ & $88 \%$ & $\sim 3 \%$ & $8 \times 10^{-11}$ \\
\hline$K_{L} \rightarrow \pi^{0} e^{+} e^{-}$ & $38 \%$ & $\sim 15 \%$ & $3.5 \times 10^{-11}$ \\
\hline$K_{L} \rightarrow \pi^{0} \mu^{+} \mu^{-}$ & $28 \%$ & $\sim 30 \%$ & $1.5 \times 10^{-11}$ \\
\hline
\end{tabular}

Table 1: Short-distance sensitivity of the four "golden channels" in the Kaon sector. The second column stands for the contribution to the total rate arising from electroweak dynamics while the third column stands for the irreducible error in the short-distance amplitude.

In the kaon sector, it is typically difficult to keep long-distance effects within a sufficiently accurate level to perform precise tests of short-distance dynamics. This happens in the four golden modes $K_{L} \rightarrow \pi^{0} v \bar{v}, K^{+} \rightarrow \pi^{+} v \bar{v}, K_{L} \rightarrow \pi^{0} e^{+} e^{-}$, and $K_{L} \rightarrow \pi^{0} \mu^{+} \mu^{-}$, which represent a unique window on $s \rightarrow d$ FCNC transitions [12]. As shown in Table 1, the theoretical cleanness of these four modes is not the same. The two neutrino channels are exceptionally clean and their decay rates have been computed with a unique accuracy, much beyond that reached by any other FCNC process in the $B$ and $K$ systems [13].

\subsection{SM predictions}

$\underline{K \rightarrow \pi v \bar{v}}$ The unique theoretical cleanness of $K \rightarrow \pi v \bar{v}$ decays within the SM arises from the fact that these processes are loop induced (of $O\left(G_{F}^{2}\right)$ ) by means of a power-like GIM mechanism (largely) dominated by top-quark loops. This implies a severe suppression of non-perturbative effects $[16,14,15]$. In contrast, typical loop-induced processes governed by gluon (photon) penguins are of $O\left(G_{F} \alpha_{s}\right)\left(O\left(G_{F} \alpha_{\mathrm{em}}\right)\right)$ and the corresponding GIM mechanism is of logarithmic-type which results in a much less severe suppression of long-distance effects.

Moreover, both in the SM and in MFV models, the leading contributions to $K \rightarrow \pi v \bar{v}$ amplitudes can be described by a single dimension-six effective operator,

$$
Q_{s d}^{v v}=\bar{s} \gamma^{\mu}\left(1-\gamma_{5}\right) d \bar{v} \gamma_{\mu}\left(1-\gamma_{5}\right) \nu
$$

The hadronic matrix elements of $Q_{s d}^{v v}$ relevant to $K \rightarrow \pi v \bar{v}$ amplitudes can be extracted directly from the well-measured $K \rightarrow \pi e v$ decays, including isospin breaking corrections [17].

Within the SM, the main theoretical error in the evaluation of the $K^{+} \rightarrow \pi^{+} v \bar{v}$ amplitude comes from the subleading, but still sizable, charm contribution. This theoretical error became safely negligible after the NNLO QCD calculation [18] of the charm contribution to the Wilson coefficient of $Q_{s d}$.

The error associated to non-perturbative effects around and below the charm scale (dimensioneight operators and light-quark loops) has also been quantified and reduced [15]. As a result, the present updated prediction for the charged channel reads:

$$
\mathscr{B}\left(K^{+} \rightarrow \pi^{+} v \bar{v}\right)=(8.2 \pm 1.0) \times 10^{-11} .
$$



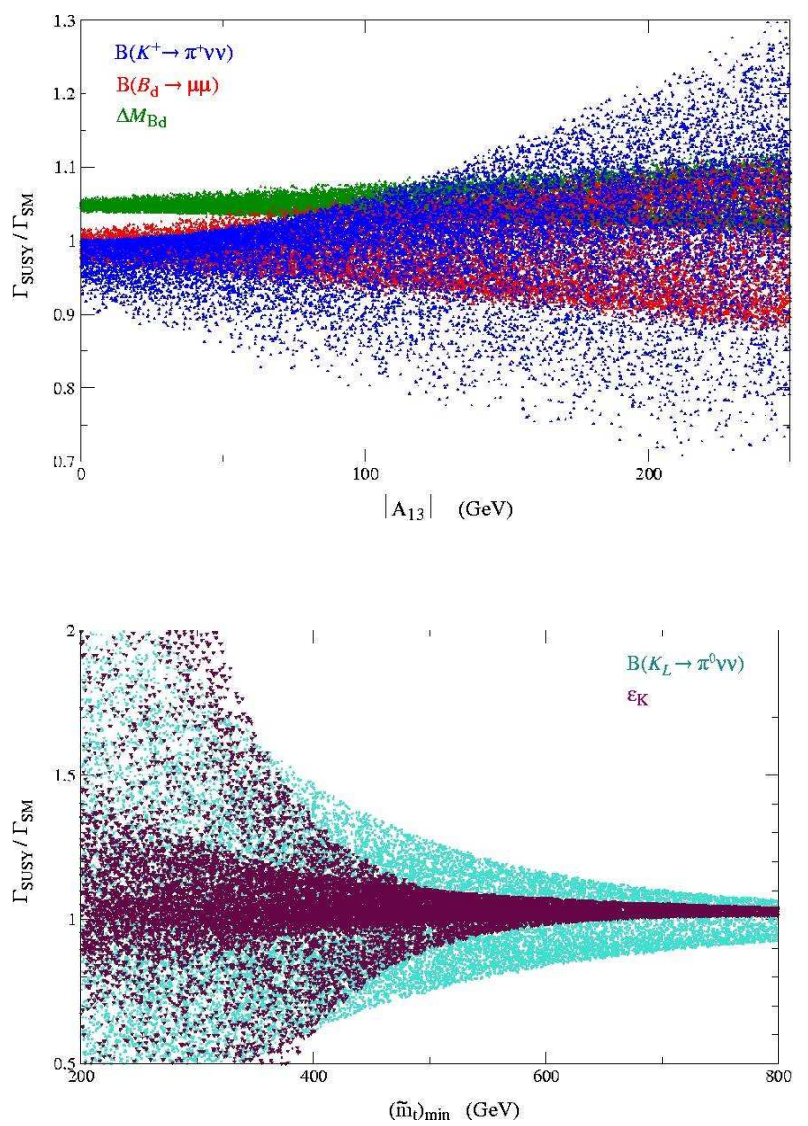

Figure 3: Dependence of various FCNC observables (normalized to their SM value) on the up-type trilinear terms $\left(A_{13}\right)$ and on squark masses in the general MSSM [24] Upper plot: $\mathscr{B}\left(K^{+} \rightarrow \pi^{+} v \bar{v}\right)$ (blue/dark gray), $\mathscr{B}\left(B_{d} \rightarrow \mu^{+} \mu^{-}\right)$(red/gray lower-region), $\Delta M_{B_{d}}$ (green/gray upper-region) as a function of $A_{13}$. Lower plot: $\varepsilon_{K}$ (bordeaux/dark gray) and $\mathscr{B}\left(K_{L} \rightarrow \pi^{0} v \bar{v}\right)$ (light blue/light gray) as a function of the lightest up-type squark mass.

Theoretically, the $K_{L} \rightarrow \pi^{0} v \bar{v}$ process is even cleaner [19]. In fact, here only the CP-violating part of the dimension-six effective Hamiltonian (where the charm contribution is safely negligible) contributes to $K_{2} \rightarrow \pi^{0} v \bar{v}$. Intermediate and long-distance effects in this process are confined only to the indirect-CP-violating contribution [20] and to the CP-conserving one [16], which are both extremely small. This enables us to write an expression for the $K_{L} \rightarrow \pi^{0} v \bar{v}$ rate in terms of short-distance parameters

$$
\begin{aligned}
& \mathscr{B}\left(K_{L} \rightarrow \pi^{0} v \bar{v}\right)_{\mathrm{SM}}=4.16 \times 10^{-10} \\
& \times\left[\frac{\bar{m}_{t}\left(m_{t}\right)}{167 \mathrm{GeV}}\right]^{2.30}\left[\frac{\mathfrak{J}\left(V_{t s}^{*} V_{t d}\right)}{\lambda^{5}}\right]^{2}
\end{aligned}
$$

which has a theoretical error below $3 \%$. 


\subsection{Rare $K$ decays beyond the SM}

The short distance nature of the $s \rightarrow d v \bar{v}$ transition is the reason its strong sensitivity to possible NP effects [21]. Observable deviations from the SM predictions are expected in many specific frameworks. In particular, large effects are expected in models with non-MFV structures, such as scenarios with enhanced Z-penguins [22], the MSSM with non-MFV soft-breaking terms [23, 24, 25] or with $R$-parity violation [26]. The effects are much smaller in models which respect the MFV criterion, such as the low-energy supersymmetric scenarios analyzed in Ref. [24, 27], or the little-Higgs and large-extra-dimension models discussed in Ref. [29] and Ref. [28]. Present experimental data do not allow yet to fully explore the high-discovery potential of these modes. However, it is worth to stress that the evidence of the $K^{+} \rightarrow \pi^{+} v \bar{v}$ transition obtained at BNL [30] already provides highly non-trivial constraints on the realistic scenarios with large new sources of flavour mixing.

Within a MFV framework, deviations from the SM expectations in $K \rightarrow \pi \nu \bar{v}$ are highly correlated to the spectrum of the new degrees of freedom [24], thus, even in this pessimist scenario, precise measurements of these modes would be very valuable. In presence of non-MFV structures, the two $K \rightarrow \pi v \bar{v}$ modes are usually the most sensitive probes of new sources of flavor symmetry breaking which also violates the $S U(2)_{L}$ gauge symmetry (such as the up-type trilinear terms in the MSSM, see Fig. 3). Within these general frameworks, significant new information can also be extracted from the $K_{L} \rightarrow \pi^{0} \ell^{+} \ell^{-}$modes [22, 24].

\section{Conclusions}

Kaon physics has played a crucial role in the past in the understanding of the flavour structure of the SM. However, it is worthwhile to stress that kaon observables are expected to play a very important role also in the future, when we will deal with the attempt to reconstruct the flavour sector of the NP scenario that will hopefully emerge at the LHC. In this document, we focused on particularly interesting kaon observables as $R_{K}=\Gamma\left(K_{e 2}\right) / \Gamma\left(K_{\mu 2}\right)$ and the rare kaon decays $K \rightarrow \pi \nu \bar{v}$. The former represents among the most sensitive probes of Lepton Universality breaking effects arising in NP scenarios like Supersymmetry; the latter are of special importance to probe the flavour structure of beyond SM theories and hence to address and understand the flavor problem in the quark sector.

\section{References}

[1] W.J. Marciano and A. Sirlin, Phys.Rev.Lett. 713629 (1993); M.Finkemeier, Phys.Lett. B 387391 (1996); V. Cirigliano and I. Rosell, Phys. Rev. Lett. 99 (2007) 231801.

[2] C. Amsler et al. (PDG), Phys. Lett. B667 (2008) 1.

[3] F. Ambrosino et al., arXiv:0907.3594.

[4] E. Goudzovski, these proceedings.

[5] A. Masiero, P. Paradisi and R. Petronzio, Phys. Rev. D74 (2006) 011701; JHEP 0811 (2008) 42.

[6] W.S.Hou, Phys. Rev. D 48, 2342 (1992). 
[7] L. J. Hall, R. Rattazzi and U. Sarid, Phys. Rev. D 50, 7048 (1994).

[8] K. S. Babu and C. Kolda, Phys. Rev. Lett. 89 (2002) 241802 [hep-ph/0206310].

[9] G. Anderson, S. Raby, S. Dimopoulos, L. J. Hall and G. D. Starkman, Phys. Rev. D 49 (1994) 3660 [hep-ph/9308333].

[10] G. Isidori and P. Paradisi, Phys. Lett. B 639 (2006) 499 [hep-ph/0605012].

[11] J. R. Ellis, L. Roszkowski and Z. Lalak, Phys. Lett. B 245 (1990) 545.

[12] M. Artuso et al., Eur. Phys. J. C57 (2008) 309.

[13] D. Bryman, A. J. Buras, G. Isidori and L. Littenberg, Int. J. Mod. Phys. A 21, 487 (2006) [hep-ph/0505171]; A. J. Buras, F. Schwab and S. Uhlig, Rev. Mod. Phys. 80 (2008) 965 [arXiv:hep-ph/0405132].

[14] A. F. Falk, A. Lewandowski and A. A. Petrov, Phys. Lett. B 505, 107 (2001) [hep-ph/0012099].

[15] G. Isidori, F. Mescia and C. Smith, Nucl. Phys. B 718, 319 (2005) [hep-ph/0503107].

[16] G. Buchalla and G. Isidori, Phys. Lett. B 440, 170 (1998) [hep-ph/9806501]; D. Rein and L.M. Sehgal, Phys. Rev. D 39, 3325 (1989).

[17] W.J. Marciano and Z. Parsa, Phys. Rev. D 53, R1 (1996).

[18] A. J. Buras, M. Gorbahn, U. Haisch and U. Nierste, Phys. Rev. Lett. 95 (2005) 261805 [arXiv:hep-ph/0508165]; A. J. Buras, M. Gorbahn, U. Haisch and U. Nierste, JHEP 0611 (2006) 002 [arXiv:hep-ph/0603079].

[19] L. Littenberg, Phys. Rev. D39 (1989) 3322.

[20] G. Buchalla and A. J. Buras, Phys. Rev. D 54, 6782 (1996) [hep-ph/9607447].

[21] Y. Grossman and Y. Nir, Phys. Lett. B 398, 163 (1997) [hep-ph/9701313].

[22] G. Colangelo and G. Isidori, JHEP 9809, 009 (1998) [hep-ph/9808487]; A.J. Buras and L. Silvestrini, Nucl. Phys. B 546, 299 (99) [hep-ph/9811471]; G. Buchalla, G. Hiller and G. Isidori Phys. Rev. D 63, 014015 (2001) [hep-ph/0006136]. A. J. Buras, R. Fleischer, S. Recksiegel and F. Schwab, Phys. Rev. Lett. 92 (2004) 101804.

[23] A. J. Buras, T. Ewerth, S. Jager and J. Rosiek, Nucl. Phys. B 714, 103 (2005) [hep-ph/0408142].

[24] G. Isidori, F. Mescia, P. Paradisi, C. Smith and S. Trine, JHEP 0608 (2006) 064 [arXiv:hep-ph/0604074].

[25] G. Isidori and P. Paradisi, Phys. Rev. D 73 (2006) 055017 [hep-ph/0601094].

[26] A. Deandrea, J. Welzel and M. Oertel, JHEP 0410, 038 (2004) [hep-ph/0407216].

[27] A. J. Buras et al. Nucl. Phys. B592 (2001) 55 [hep-ph/0007313].

[28] A. J. Buras, M. Spranger and A. Weiler, Nucl. Phys. B 660, 225 (2003) [hep-ph/0212143].

[29] M. Blanke, A. J. Buras, A. Poschenrieder, S. Recksiegel, C. Tarantino, S. Uhlig and A. Weiler, JHEP 0701 (2007) 066 [arXiv:hep-ph/0610298]; T. Goto, Y. Okada and Y. Yamamoto, Phys. Lett. B 670 (2009) 378 [arXiv:0809.4753 [hep-ph]].

[30] S. Adler et al. [E787 Collaboration], Phys. Rev. Lett. 79 (1997) 2204; ibid. 84 (2000) 3768; Phys. Rev. Lett. 88, (2002) 041803. A. V. Artamonov et al. [E949 Collaboration], hep-ex/0403036. 